\title{
Distribution of delirium motor subtypes in the intensive care unit: a systematic scoping review
}

\author{
Kirstine N. la Cour ${ }^{1 *} \mathbb{D}$, Nina C. Andersen-Ranberg ${ }^{1}$, Sarah Weihe ${ }^{1}$, Lone M. Poulsen ${ }^{1}$, Camilla B. Mortensen ${ }^{1}$,
} Cilia K. W. Kjer ${ }^{1}$, Marie O. Collet ${ }^{2}$, Stine Estrup ${ }^{1}$ and Ole Mathiesen ${ }^{1,3}$

\begin{abstract}
Background: Delirium is the most common cerebral dysfunction in the intensive care unit (ICU) and can be subdivided into a hypoactive, hyperactive, or mixed motor subtype based on the clinical manifestation. The aim of this review was to describe the distribution, pharmacological interventions, and outcomes of delirium motor subtypes in ICU patients.
\end{abstract}

Methods: This systematic scoping review was performed according to the PRISMA-ScR and Cochrane guidelines. We performed a systematic search in six major databases to identify relevant studies. A meta-regression analysis was performed where pooled estimates with 95\% confidence intervals were computed by a random effect model.

Results: We included 131 studies comprising 13,902 delirious patients. There was a large between-study heterogeneity among studies, including differences in study design, setting, population, and outcome reporting. Hypoactive delirium was the most prevalent delirium motor subtype (50.3\% [95\% Cl 46.0-54.7]), followed by mixed delirium (27.7\% [95\% Cl 24.1-31.3]) and hyperactive delirium (22.7\% [95\% Cl 19.0-26.5]). When comparing the delirium motor subtypes, patients with mixed delirium experienced the longest delirium duration, ICU and hospital length of stay, the highest ICU and hospital mortality, and more frequently received administration of specific agents (antipsychotics, a2-agonists, benzodiazepines, and propofol) during ICU stay. In studies with high average age for delirious patients (>65 years), patients were more likely to experience hypoactive delirium.

Conclusions: Hypoactive delirium was the most prevalent motor subtype in critically ill patients. Mixed delirium had the worst outcomes in terms of delirium duration, length of stay, and mortality, and received more pharmacological interventions compared to other delirium motor subtypes. Few studies contributed to secondary outcomes; hence, these results should be interpreted with care. The large between-study heterogeneity suggests that a more standardized methodology in delirium research is warranted.

Keywords: Critically ill, Delirium, Delirium motor subtype, Intensive care

*Correspondence: klac@regionsjaelland.dk

${ }^{1}$ Department of Anesthesiology, Centre for Anaesthesiological Research, Zealand University Hospital, Koege, Denmark

ESICM 34th Annual Congress, LIVES 2021 Digital, 3-6 October, Copenhagen

Full list of author information is available at the end of the article

\section{Introduction}

Delirium is a cerebral dysfunction affecting nearly onethird of patients in the intensive care unit (ICU) $[1,2]$. The clinical manifestations of delirium exhibit great variance. Lipowski et al. [3] were the first to suggest the use of hyperactive and hypoactive motor subtypes to original author(s) and the source, provide a link to the Creative Commons licence, and indicate if changes were made. The images or other third party material in this article are included in the article's Creative Commons licence, unless indicated otherwise in a credit line to the material. If material is not included in the article's Creative Commons licence and your intended use is not permitted by statutory regulation or exceeds the permitted use, you will need to obtain permission directly from the copyright holder. To view a copy of this licence, visit http://creativecommons.org/licenses/by/4.0/. The Creative Commons Public Domain Dedication waiver (http://creativeco mmons.org/publicdomain/zero/1.0/) applies to the data made available in this article, unless otherwise stated in a credit line to the data. 
describe the different manifestations, and with the term mixed delirium being introduced shortly after [4].

A patient with hyperactive delirium is in an agitated state of mind and may display symptoms of aggression and restlessness, whereas a patient with hypoactive delirium is in an apathetic state of mind and may display symptoms of lethargy, depression, and stupor. In mixed delirium, the patient shifts between hyperactive and hypoactive delirium. All delirium motor subtypes experience elements of the hallmark symptoms in delirium such as confusion and inattention [5]. Delirium may also be divided into other clinical phenotypes based on underlying mechanisms, e.g., sedation- or hypoxic-associated delirium [6].

We still have limited knowledge about the pathophysiology of delirium. The Neurotransmitter Hypothesis suggests that specific neurotransmitter interactions with the cholinergic pathway might determine delirium motor subtype [7-9]. Currently no evidence-based pharmacological treatment exists for this severe condition $[1,10]$. Delirium in the ICU is associated with increased shortand long-term impairments [2, 11-13]. The extent of impairments and whether they are associated with delirium motor subtypes are still to be determined. Recently, hypoactive delirium was associated with the poorest prognosis of survival compared with mixed and hyperactive delirium [14, 15], whereas another study found that the duration of the different motoric subtypes was not associated with long-term functional outcomes [16]. Considering the heterogeneity in etiology, manifestation, treatment response, and outcomes of the three delirium motor subtypes, it has been speculated that these in essence represent different disease entities [17, 18].

A key to future treatment strategies may depend on increased knowledge of distribution and current treatments of the delirium motor subtypes. In this scoping review, we therefore aimed to describe distribution, pharmacological interventions, and outcomes associated with delirium motor subtypes in adult critically ill patients.

\section{Methods}

This review was conducted in accordance with Preferred Reporting Items for Systematic reviews and Meta-Analyses extension for Scoping Reviews (PRISMA-ScR) [19] and preregistered the 26th of September 2017 at PROSPERO (ID:CRD42017076503). The full protocol is available from the corresponding author on request.

\section{Literature search}

The literature search strategy was designed in collaboration with a Health Sciences Librarian with expertise in systematic reviews and was performed twice, with the last search in April 2021 in the following databases:
Cochrane Central Register of Controlled Trials (CENTRAL), MEDLINE/PubMed, Ovid/EMBASE, Cumulative Index to Nursing \& Allied Health Literature (CINAHL), PsycINFO and Google Scholar. We manually screened ClinicalTrials.gov, EU Clinical Trial Registry, FDA Trial registry, reference lists and systematic reviews for eligible studies. Additional file shows the detailed search strings (see Additional file 1: Search strings).

\section{Eligibility}

We included randomized clinical trials (RCTs), quasirandomized studies, and observational studies with no restrictions to language, publication date or journal, and meeting the following criteria: Patients of $\geq 18$ years of age, admission to an intensive care unit (ICU), and presenting data on delirium motor subtype distribution. Exclusion criteria were: no full text obtainable (abstracts only, preprints, and conference proceedings were excluded), studies only including patients with delirium tremens (alcohol withdrawal delirium), and studies conducted in a post-anesthesia care unit.

\section{Delirium and motor subtype definition}

The diagnosis of delirium was determined by methods as described in the included studies (see Additional file 1: Table S2). For studies using more than one assessment method/tool (e.g., in studies comparing two or more tools), we prioritized the results as follows: 1 . Diagnostic and Statistical Manual for Mental Disorders (DSM III-V), 2. The Confusion Assessment Method for the ICU (CAM-ICU), and 3. Intensive Care Delirium Screening Checklist (ICDSC). Definition of the delirium motor subtype was also determined by methods as described in the included studies, whether these were based on sedation/agitation scales (such as Richmond Agitation and Sedation Scale (RASS)) or the clinical presentation of the patient.

\section{Study selection and data extraction}

Six authors (KC, NA, LMP, SW, MOC, and CBM), working in pairs, screened titles and abstracts, and extracted data from included full text studies. Disagreements were solved by consensus or by involving OM. We used EndNote version X9, Covidence (https://www.covidence. org), and Microsoft Excel to manage records, data, and de-duplicate references. Extracted data are listed in Additional file (see Additional file 1: List of extracted data). For missing or incomplete information, we contacted the corresponding authors by email. 


\section{Outcome measures}

Our primary outcome was the distribution of delirium motor subtypes. Distribution was defined as the proportion of hypoactive, hyperactive, and mixed delirium in the pooled delirious cohort. Mixed delirium was defined as any combination of hypo- and hyperactive delirium assessments during ICU stay. We did not distinguish between incidence and prevalence when assessing the distribution of delirium motor subtypes. Delirium incidence and prevalence for the pooled cohort were secondary outcomes. We defined the incidence of delirium as being newly developed delirium in the ICU and prevalence of delirium as being all diagnosed delirium cases in the ICU. We performed a sensitivity analysis comparing incidence and prevalence of delirium between studies including versus excluding comatose patients. For each delirium motor subtype, we reported the following secondary outcomes: delirium duration, pharmacological interventions, hospital and ICU length of stay, and ICU and hospital mortality. Studies mainly reported unspecific delirium treatment strategies, such as following the PADIS guideline [20]. We were unable to retrieve data on specific agents used for delirium treatment; however, some studies reported a pharmacological treatment strategy for delirium and specific agents given to delirious patients during ICU, but with no indication reported. Consequently, we reported pharmacological interventions in terms of delirium targeted pharmacological strategy, defined as number of patients intervened with a pharmacological delirium treatment strategy, and administration of specific agents defined as reported administration of antipsychotics, $\alpha 2$-agonists, benzodiazepines, and propofol with no limits to indication. We chose these agents as antipsychotics, $\alpha 2$-agonists, and benzodiazepines, which are the most commonly used agents, for delirium treatment [21]. Propofol was added since this agent is frequently used to sedate delirious patients. We excluded RCTs in the analyses of pharmacological interventions.

\section{Critical appraisal}

We used Cochrane Collaboration Risk of Bias Tool [22] to evaluate the risk of systematic errors of included RCTs as either high, unclear, or low risk of bias. For evaluation of the quality of included observational studies, we used the National Institutes of Health (NIH) Quality Assessment Tools [23] with adjudication of either poor, unclear, or good quality.

\section{Statistical analysis}

For analytic reasons, we converted values reported in medians and range or interquartile range to mean and standard deviation values according to Luo et al. and Shi et al. [24, 25]. The pooled estimates of primary and secondary outcomes were computed with meta-regression analysis using a random effect model and reported with corresponding $95 \%$ confidence intervals. The reported proportions were untransformed. Betweenstudy variance was determined with the restricted maximum-likelihood estimator. We created forest plots and used $\mathrm{I}^{2}$ statistic to evaluate between-study heterogeneity and Cochrane's Q test to establish significance of heterogeneity [26]. To evaluate differences in delirium incidence and prevalence in studies excluding and including comatose patients, we performed a sensitivity analysis. Coma was defined as stated in studies. To address confounding variables and effect modifiers, we performed a pre-planned subgroup analysis with metaregression and stratification to estimate whether the distribution of delirium motor subtypes was dependent on age, type of ICU admission, mechanical ventilation, disease severity, inclusion/exclusion of comatose patients, or RoB/quality assessment (QA). The disease severity was divided into high or low severity (high disease severity was a priori defined as a predicted risk of mortality of $\geq 50 \%$ : APACHE II $\geq 21$, SAPS II $\geq 55$, SAPS III $\geq 70$, SOFA score $\geq 11$ ). If a study reported multiple severity of illness scores, the study was rated corresponding to the score predicting the highest mortality. For subgroup analysis, we assumed a common between-study variance component. All statistical analyses were performed in R Statistical Software (https:// www.r-project.org/) version 4.0.1. [27] using the metaand metafor package.

\section{Results}

\section{Search and study characteristics}

Our initial literature search identified 18,602 studies. After screening and full text review, a total of 131 studies were included comprising a pooled cohort of 50,232 patients. We mainly excluded studies due to the lack of data on delirium motor subtype distribution or no full text obtainable (Fig. 1). Included studies differentiated notably in study design, setting, population, and outcome reporting. Randomized controlled studies (RCTs) and case-control studies typically did not report on delirium incidence nor prevalence. Characteristics of included studies are presented in Table 1. We contacted 125 authors via email, 37 answered, and 19 provided additional data. Characteristics of the total pooled cohort (see Additional file 1: Table S3) as well as an overview of included studies (see Additional file 1: Table S1) can be found in Additional file. 


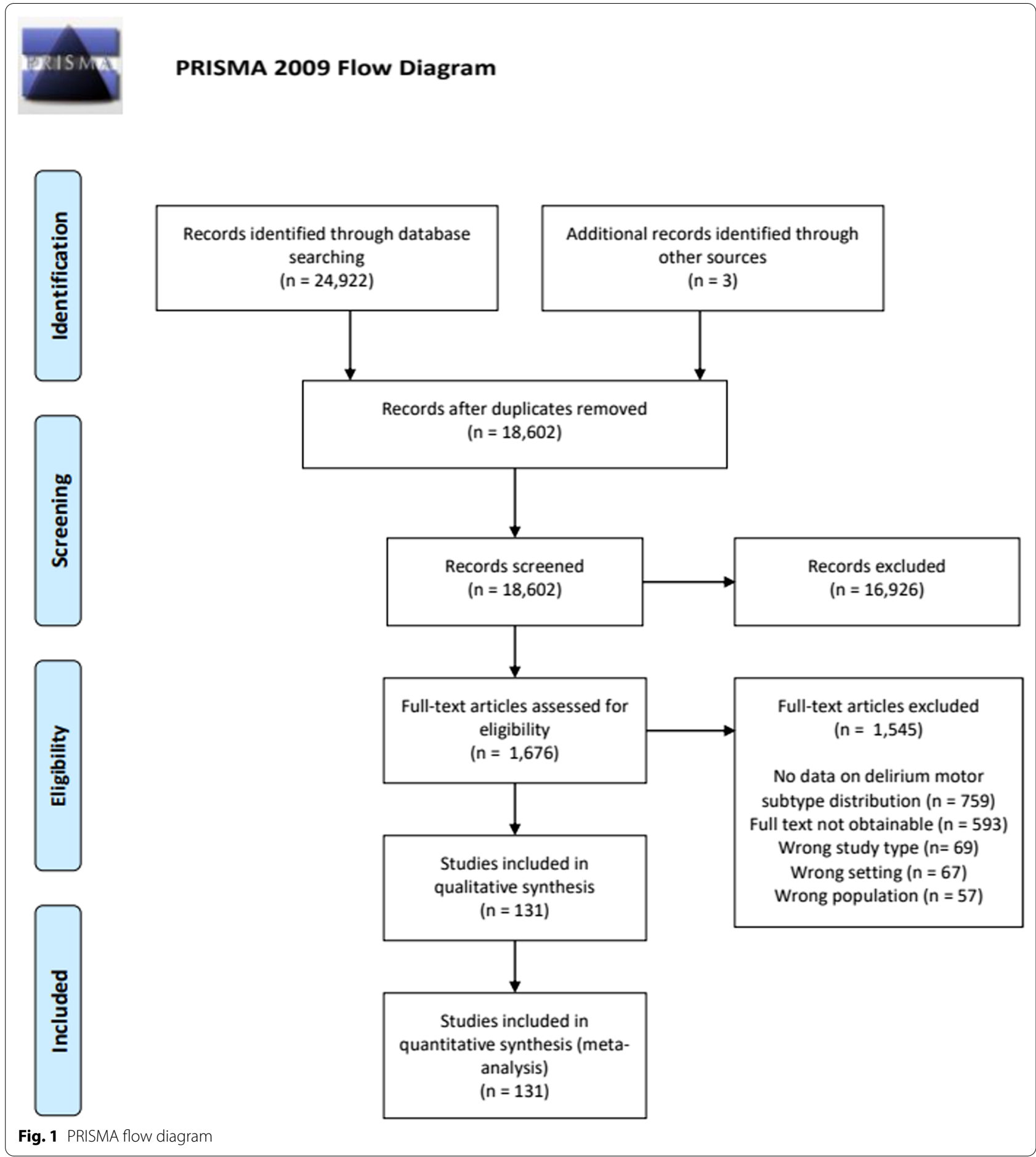

\section{Delirium incidence and prevalence}

The pooled incidence of delirium was $22.2 \%$ [95\% CI 18.6-25.8], and the pooled prevalence was 38.3\% [95\% CI 34.2-42.4]. Forrest plots in Additional file show delirium incidence and prevalence across included studies (see Additional file 1: Figs. S1 and S2). To diagnose delirium,
CAM-ICU was the most frequently used method (87.0\%) followed by DSM-III-V (18.3\%) and ICDSC (12.9\%) and most studies defined motor subtype with RASS (86.3\%). Some studies used more than one tool. On average, patients were assessed for delirium 1.7 times a day, and in most studies delirium assessments were performed 
Table 1 Characteristics of included studies

\begin{tabular}{lc}
\hline Study characteristics & No. of studies, $\boldsymbol{n}$ (\%) \\
\hline Total number of included studies & $131(100)$ \\
Study design & \\
Prospective cohort study & $96(73.3)$ \\
Retrospective cohort study & $14(10.7)$ \\
Case-control study & $6(4.6)$ \\
Randomized controlled trial & $9(6.9)$ \\
Other* & $6(4.6)$ \\
Publication year & \\
2001-2005 & $2(1.5)$ \\
2006-2010 & $15(11.5)$ \\
2011-2015 & $42(32.1)$ \\
2016-2020 & $72(55.0)$ \\
Type of ICU & \\
Surgical & $23(17.6)$ \\
Medical & $12(9.2)$ \\
Mixed & $53(40.5)$ \\
Cardiac & $35(26.7)$ \\
Other & $8(6.1)$ \\
Cochrane Risk of bias/NIH quality** & \\
High/poor & $58(44.3)$ \\
Unclear/unclear & $39(29.8)$ \\
Low/good & $34(26.0)$ \\
\hline CT &
\end{tabular}

$R C T$ randomized clinical trial, ICU intensive care unit

*Before/after studies and quasi-randomized studies

${ }^{*}$ Cochrane RoB was used for RCTs and NIH quality assessment tools were used for observational studies

by nurses throughout ICU stay (see Additional file 1: Table S2). In the sensitivity analyses comparing studies including versus excluding comatose patients, we found no significant difference in prevalence and incidence of delirium.

\section{The delirious patient cohort}

The pooled delirious cohort comprised of 13,902 patients. The mean age of patients with delirium was 66.9 years [95\% CI 65.4-68.5]. Disease severity scores were primarily reported by APACHE II score (44.3\%), and the mean score for the delirious cohort was 18.9 [95\% CI 17.5-20.3]. Patients with delirium were intervened with a delirium-targeted pharmacological strategy in $66.1 \%$ [95\% CI 55.4-76.8] of cases. The investigated pharmacological agent that was most frequently administrated to delirious patients was antipsychotics $(49.6 \%$ [95\% CI $39.2-60.0]$ ) followed by propofol $(42.4 \%$ [95\% CI $28.7-$ 56.2]), benzodiazepines (39.8\% [95\% CI 31.2-48.5]), and $\alpha 2$-agonists (26.3\% [95\% CI 17.4-35.1]). The mean length of stay for patients with delirium was 9.2 days [95\% CI 8.0-10.4] in the ICU and 19.8 days [95\% CI 17.6-22.1] in the hospital. The ICU mortality for the delirious cohort was $17.0 \%$ [95\% CI 12.6-21.4], and the hospital mortality was $21.3 \%$ [ $95 \%$ CI $14.0-28.6$ ]. Table 2 presents the characteristics of the pooled delirious cohort.

\section{Distribution of delirium motor subtypes}

One hundred and twelve studies contributed to the distribution of all three motor subtypes, while 19 studies contributed to the distribution of hypoactive and hyperactive delirium only. Dichotomous distribution of delirium motor subtypes was typically reported in studies determining delirium motor subtype upon study inclusion making it impossible to determine the mixed subtype. Among studies reporting on all three motor subtypes, we found that hypoactive delirium was the most prevalent (50.3\% [95\% CI 46.0-54.7]), followed by mixed delirium (27.7\% [95\% CI 24.1-31.3]) and hyperactive delirium (22.7\% [95\% CI 19.0-26.5]). Data on distribution of delirium motor subtypes are presented in Table 3. Forrest plots in Additional file illustrate the prevalence of the three motor subtypes across studies (see Additional file 1: Figs. S3, S4, and S5).

\section{Secondary outcomes}

Few studies reported on secondary outcomes at motor subtype level and hence contributed to these results. Patients with mixed delirium motor subtype had the longest delirium duration (days) (mixed: 3.6 [95\% CI 2.6-4.5], hypo: 2.4 [95\% CI 1.9-2.8], hyper: 2.2 [95\% CI 1.7-2.7]), ICU length of stay (days) (mixed: 10.3 [95\% CI 7.7-12.9], hypo: 8.4 [95\% CI 6.4-10.5], hyper: 6.9 [95\% CI 5.1-8.8]), and hospital length of stay (days) (mixed: 25.1 [95\% CI 18.3-31.9], hypo: 20.0 [95\% CI 15.8-24.2], hyper: 18.5 [95\% CI 14.2-22.8]), and the highest ICU mortality (\%) (mixed: 30.0 [95\% CI 14.1-45.9], hypo: 27.9 [95\% CI 17.5-38.5], hyper: 21.8 [95\% CI 9.4-34.2]), and hospital mortality (\%) (mixed: 32.8 [95\% CI 15.3-50.4], hyper: 30.1 [95\% CI 4.8-57.1], hypo: 27.2 [95\% CI 10.1-44.3]). Patients with mixed delirium were more frequently received antipsychotics (\%) (58.5 [95\% CI 34.2-82.9]), $\alpha 2$-agonists (53.7 [95\% CI 25.4-82.0]), benzodiazepines (54.1 [95\% CI 29.6-78.7]), and propofol (67.7 [39.3-96.1]) than other delirium motor subtypes. Patients with hyperactive delirium were more frequently intervened with a delirium-targeted pharmacological strategy compared to patients with mixed or hypoactive delirium (\%) $(63.7$ [95\% CI 39.8-87.5] vs. 58.3 [95\% CI 34.2-82.9] vs. 38.8 [95\% CI 17.0-60.7]). For data on secondary outcomes (delirium duration, length of stay, mortality, and pharmacological interventions), see Table 4.

\section{Subgroup analysis}

In the pre-planned subgroup analysis, we found that hypoactive delirium was significantly more 
Table 2 Characteristics of patients with delirium

\begin{tabular}{|c|c|c|c|c|}
\hline $\begin{array}{l}\text { Characteristics of patients } \\
\text { with delirium }(n=13,902)\end{array}$ & $\begin{array}{l}\text { Studies reporting on } \\
\text { outcome }(n)\end{array}$ & $\begin{array}{l}\text { Delirious patients in studies } \\
\text { reporting on outcome }(n)\end{array}$ & $\begin{array}{l}\text { Pooled mean or pooled } \\
\text { proportion }\end{array}$ & $95 \% \mathrm{Cl}$ \\
\hline Age & 96 & 12,112 & 66.9 years & $65.4-68.5$ \\
\hline Sex (male) & 91 & 11,637 & $62.1 \%$ & $60.2-64.0$ \\
\hline \multicolumn{5}{|l|}{ Severity of disease } \\
\hline APACHEII & 48 & 5897 & 18.9 & $17.5-20.3$ \\
\hline SAPSII & 10 & 1206 & 42.7 & $37.0-48.4$ \\
\hline SAPSIII & 2 & 285 & 52.2 & $33.2-71.2$ \\
\hline SOFA & 16 & 2046 & 7.8 & $6.3-9.3$ \\
\hline $\begin{array}{l}\text { Delirium-targeted pharma- } \\
\text { cological strategy* }\end{array}$ & 21 & 3979 & $66.1 \%$ & $55.4-76.8$ \\
\hline \multicolumn{5}{|c|}{ Administration of specific agents** } \\
\hline Antipsychotics & 33 & 5233 & $49.6 \%$ & $39.2-60.0$ \\
\hline a2-agonists & 24 & 2474 & $26.3 \%$ & $17.4-35.1$ \\
\hline Benzodiazepines & 38 & 3843 & $39.8 \%$ & $31.2-48.5$ \\
\hline Propofol & 18 & 2057 & $42.4 \%$ & $28.7-56.2$ \\
\hline \multicolumn{5}{|l|}{ Length of stay } \\
\hline ICU & 67 & 9813 & 9.2 days & $8.0-10.4$ \\
\hline Hospital & 42 & 7889 & 19.8 days & $17.6-22.1$ \\
\hline \multicolumn{5}{|l|}{ Mortality } \\
\hline $\mathrm{ICU}$ & 29 & 4519 & $17.0 \%$ & $12.6-21.4$ \\
\hline Hospital & 18 & 1909 & $21.3 \%$ & $14.0-28.6$ \\
\hline
\end{tabular}

APACHE Acute Physiology and Chronic Health Evaluation, SAPS Simplified Acute Physiology Score, SOFA Sequential Organ Failure Assessment

* Patients intervened with a delirium-targeted pharmacological treatment strategy during ICU stay

** Patients receiving administration of specific agents (as listed) while in the ICU with no limits to indication

Table 3 Distribution of delirium motor subtypes in studies with 3 or 2 motoric subtypes

\begin{tabular}{|c|c|c|c|c|}
\hline Delirium motor subtype & $\begin{array}{l}\text { Studies reporting on } \\
\text { outcome }(n)\end{array}$ & $\begin{array}{l}\text { Delirious patients in studies reporting } \\
\text { on outcome }(n)\end{array}$ & $\begin{array}{l}\text { Pooled proportion } \\
(\%)\end{array}$ & $95 \% \mathrm{Cl}$ \\
\hline \multicolumn{5}{|c|}{ Distribution of delirium motor subtypes } \\
\hline \multicolumn{5}{|c|}{ Studies reporting 3 motoric subtypes } \\
\hline Hypoactive* & 111 & 11,663 & 50.3 & $46.0-54.7$ \\
\hline Hyperactive ${ }^{* *}$ & 109 & 11,626 & 22.7 & $19.0-26.5$ \\
\hline Mixed & 108 & 11,509 & 27.7 & $24.1-31.3$ \\
\hline \multicolumn{5}{|c|}{ Studies reporting 2 motoric subtypes ${ }^{* * *}$} \\
\hline Hypoactive & 19 & 2434 & 61.4 & $49.2-73.5$ \\
\hline Hyperactive & 19 & 2434 & 38.6 & $26.5-50.8$ \\
\hline
\end{tabular}

* Three studies only had data on distribution of the hypoactive delirium motor subtype

** One study only had data on distribution of the hyperactive delirium motor subtype

${ }^{* * *}$ These studies only discriminated between hypoactive and hyperactive delirium motor subtype

prevalent in studies with older delirious patients (mean age $\geq 65$ years) compared to studies with younger delirious patients (mean age $<65$ years) (\%) (54.0 [95\% CI 48.5-59.5] vs. 40.0 [95\% CI 32.7-47.2]) $(p=0.002)$. Mixed delirium was significantly more prevalent in studies with younger delirious patients (mean age $<65$ years) compared to studies with older delirious patients (mean age $\geq 65$ years) (\%) $(35.8[95 \%$ CI 29.0-42.6] vs. 23.8 [95\% CI 18.7-28.9]) $(p=0.006)$. The prevalence of mixed delirium was significantly higher amongst studies with low risk of bias or good quality compared to studies with high risk of bias or poor quality (\%) (33.4 [95\% CI 26.1-40.7] vs. 23.4 [95\% CI 17.8-29.0]) $(p=0.03)$. Distribution of delirium 
Table 4 Secondary outcomes

\begin{tabular}{|c|c|c|c|c|}
\hline Delirium motor subtype & $\begin{array}{l}\text { Studies reporting on outcome } \\
(n)\end{array}$ & $\begin{array}{l}\text { No. of patients with outcome } \\
(n)\end{array}$ & Pooled mean/proportion & $95 \% \mathrm{Cl}$ \\
\hline \multicolumn{5}{|l|}{ Delirium duration } \\
\hline Hypoactive & 26 & 1589 & 2.4 days & $1.9-2.8$ \\
\hline Hyperactive & 25 & 709 & 2.2 days & $1.7-2.7$ \\
\hline Mixed & 23 & 1591 & 3.6 days & $2.6-4.5$ \\
\hline \multicolumn{5}{|l|}{ ICU length of stay } \\
\hline Hypoactive & 25 & 1707 & 8.4 days & $6.4-10.5$ \\
\hline Hyperactive & 22 & 541 & 6.9 days & $5.1-8.8$ \\
\hline Mixed & 22 & 1513 & 10.3 days & $7.7-12.9$ \\
\hline \multicolumn{5}{|l|}{ Hospital length of stay } \\
\hline Hypoactive & 19 & 1596 & 20.0 days & $15.8-24.2$ \\
\hline Hyperactive & 16 & 545 & 18.5 days & $14.2-22.8$ \\
\hline Mixed & 17 & 1670 & 25.1 days & $18.3-31.9$ \\
\hline \multicolumn{5}{|l|}{ ICU mortality } \\
\hline Hypoactive & 12 & 779 & $27.9 \%$ & $17.5-38.3$ \\
\hline Hyperactive & 9 & 313 & $21.8 \%$ & $9.4-34.2$ \\
\hline Mixed & 9 & 685 & $30.0 \%$ & $14.1-45.9$ \\
\hline \multicolumn{5}{|l|}{ Hospital mortality } \\
\hline Hypoactive & 4 & 305 & $27.2 \%$ & $10.1-44.3$ \\
\hline Hyperactive & 3 & 106 & $30.1 \%$ & $4.8-57.1$ \\
\hline Mixed & 4 & 305 & $32.8 \%$ & $15.3-50.4$ \\
\hline \multicolumn{5}{|c|}{ Delirium-targeted pharmacological strategy* } \\
\hline Hypoactive & 7 & 358 & $38.8 \%$ & $17.0-60.7$ \\
\hline Hyperactive & 7 & 258 & $63.7 \%$ & $39.8-87.5$ \\
\hline Mixed & 7 & 479 & $58.3 \%$ & $32.4-84.3$ \\
\hline \multicolumn{5}{|c|}{ Administration of antipsychotics ${ }^{* *}$} \\
\hline Hypoactive & 9 & 885 & $39.4 \%$ & $16.0-62.9$ \\
\hline Hyperactive & 8 & 321 & $56.2 \%$ & $31.6-80.8$ \\
\hline Mixed & 9 & 1073 & $58.5 \%$ & $34.2-82.9$ \\
\hline \multicolumn{5}{|c|}{ Administration of a2-agonists** } \\
\hline Hypoactive & 7 & 214 & $22.1 \%$ & $10.1-34.1$ \\
\hline Hyperactive & 7 & 188 & $33.4 \%$ & $13.5-53.3$ \\
\hline Mixed & 7 & 275 & $53.7 \%$ & $25.4-82.0$ \\
\hline \multicolumn{5}{|c|}{ Administration of benzodiazepines ${ }^{* *}$} \\
\hline Hypoactive & 7 & 237 & $31.9 \%$ & $16.5-47.2$ \\
\hline Hyperactive & 7 & 194 & $28.0 \%$ & $21.8-34.1$ \\
\hline Mixed & 7 & 251 & $54.1 \%$ & $29.6-78.7$ \\
\hline \multicolumn{5}{|l|}{ Administration of propofol** } \\
\hline Hypoactive & 6 & 188 & $53.1 \%$ & $31.0-75.2$ \\
\hline Hyperactive & 6 & 164 & $56.0 \%$ & $28.1-83.8$ \\
\hline Mixed & 6 & 242 & $67.7 \%$ & $39.3-96.1$ \\
\hline
\end{tabular}

* Patients intervened with a delirium-targeted pharmacological treatment strategy during ICU stay

${ }^{*}$ Patients receiving administration of this specific agents while in the ICU with no limits to indication

motor subtypes was not dependent on the type of ICU (medical vs. surgical and cardiac vs. other ICUs), mechanical ventilation, including vs. excluding comatose patients or severity of disease. Table 5 presents the subgroup analysis.

\section{Discussion}

In this scoping systematic review comprising 131 studies and a pooled cohort of 50,232 ICU patients of whom 13,902 had delirium, we found hypoactive delirium to be the most prevalent delirium motor subtype among ICU 
Table 5 Subgroup analysis

\begin{tabular}{|c|c|c|c|}
\hline Subgroup (no. of studies in this subgroup) & $\begin{array}{l}\text { Hypoactive pooled proportion (\%) } \\
(95 \% \mathrm{Cl})\end{array}$ & $\begin{array}{l}\text { Hyperactive pooled proportion (\%) } \\
(95 \% \mathrm{Cl})\end{array}$ & $\begin{array}{l}\text { Mixed pooled } \\
\text { proportion }(\%) \\
(95 \% \mathrm{Cl})\end{array}$ \\
\hline \multicolumn{4}{|l|}{ Subgroup analysis } \\
\hline \multicolumn{4}{|l|}{ Medical versus surgical ICUs } \\
\hline Medical $(n=12)$ & $40.4(24.8-55.9)$ & $28.2(15.6-40.7)$ & $29.4(17.6-41.1)$ \\
\hline Surgical $(n=23)$ & $54.1(43.9-64.3)$ & $23.7(15.3-32.1)$ & $26.3(18.3-34.2)$ \\
\hline \multicolumn{4}{|l|}{ Cardiac versus other ICUs } \\
\hline Cardiac $(n=35)$ & $51.2(43.1-60.3)$ & $28.5(21.2-35.8)$ & $21.7(14.7-28.8)$ \\
\hline Other $(n=96)$ & $50.0(44.9-55.0)$ & $20.7(16.4-25.0)$ & $29.5(25.4-33.6)$ \\
\hline \multicolumn{4}{|l|}{ Mean age } \\
\hline$<65(n=38)$ & $40.0(32.7-47.2)^{*}$ & $23.2(16.5-29.9)$ & $35.8(29.0-42.6)^{*}$ \\
\hline$\geq 65(n=60)$ & $\begin{array}{l}54.0(48.5-59.5)^{*} \\
p=0.002\end{array}$ & $22.6(17.6-27.7)$ & $\begin{array}{l}23.8(18.7-28.9)^{*} \\
p=0.006\end{array}$ \\
\hline \multicolumn{4}{|l|}{ Mechanical ventilation } \\
\hline$\leq 20 \% \mathrm{MV}(n=13)$ & $53.7(38.3-69.1)$ & $27.1(15.8-38.4)$ & $24.6(10.0-39.2)$ \\
\hline$\geq 80 \% \operatorname{MV}(n=38)$ & $48.9(40.4-57.3)$ & $17.3(11.5-23.0)$ & $32.7(25.2-40.3)$ \\
\hline \multicolumn{4}{|l|}{ Disease severity } \\
\hline Low $(n=58)$ & $51.3(45.3-57.3)$ & $21.1(16.7-25.4)$ & $29.2(23.8-34.6)$ \\
\hline $\operatorname{High}(n=21)$ & $49.8(39.3-60.3)$ & $16.9(9.5-24.4)$ & $32.1(22.7-41.4)$ \\
\hline \multicolumn{4}{|l|}{ Comatose patients } \\
\hline Including $(n=40)$ & $63.7(46.7-60.8)$ & $21.5(15.4-27.6)$ & $27.4(21.3-33.5)$ \\
\hline Excluding $(n=65)$ & $48.4(42.8-54.0)$ & $22.1(17.4-26.9)$ & $28.3(23.6-32.9)$ \\
\hline \multicolumn{4}{|l|}{ Risk of bias/quality assessment } \\
\hline Low RoB/good QA $(n=34)$ & $53.3(44.5-62.1)$ & $18.1(10.4-25.8)$ & $33.4(26.1-40.7)^{*}$ \\
\hline High RoB/Poor QA $(n=58)$ & $50.2(43.1-57.2)$ & $25.1(19.1-31.2)$ & $\begin{array}{l}23.4(17.8-29.0)^{*} \\
p=0.03\end{array}$ \\
\hline
\end{tabular}

ICU intensive care unit, $R O B$ risk of bias, $Q A$ quality assessment

* Statistically significant difference

patients accounting for half $(50.3 \%)$ of delirium cases. Furthermore, we identified patients with mixed delirium to have the longest delirium duration, ICU and hospital length of stay, and the highest ICU and hospital mortality. Data on pharmacological interventions were complex. While patients with hyperactive delirium were more frequently intervened with a delirium-targeted pharmacological strategy, patients with mixed delirium were more likely to receive administration of specific pharmacological agents (antipsychotics, $\alpha 2$-agonists, benzodiazepines, and propofol) during their ICU stay (no reported indication). Our subgroup analysis identified hypoactive delirium to be more prevalent in studies with high mean age ( $\geq 65$ years) in delirious patients and mixed delirium to be more prevalent in studies with low mean age ( $<65$ years) in delirious patients. The subgroup analyses also revealed a significantly higher proportion of mixed delirium cases in studies with low risk of bias or good quality.

Our reported distribution of delirium motor subtypes is in accordance with findings in a recent review by Krewulak et al. [28] who included 48 studies and 27,342 ICU patients. Krewulak et al. [28] found hypoactive delirium to have the highest mortality in four of seven included studies. In contrast, we found the highest ICU and hospital mortality in patients with mixed delirium in eight of twelve studies. These differences could be due to the excessive heterogeneity between the included studies. However, in one of the most recent and largest studies $(n=6323)$ reporting on delirium motor subtypes, the highest mortality rates and ICU length of stay were also found for patients with mixed delirium [29]. Pisani et al. [30] found an association between the number of days of delirium in the ICU and higher mortality. These findings support why mixed delirium with the longest delirium duration also has the highest mortality. In a recent study from the BRAIN-ICU cohort, hypoactive delirium was not associated with increased risk of death in the hospital [31]. Patients with mixed delirium received more pharmacological interventions than other delirium motor subtypes. An important question to ask is whether this 
could be associated with the increased mortality found in these patients.

Management of delirium is challenging, and no evidence-based treatment currently exists. Few studies in this review reported on pharmacological interventions between delirium motor subtypes. We found ICU patients diagnosed with hyperactive delirium to be more frequently intervened with a delirium-targeted pharmacological strategy. This is in accordance with other studies [32, 33]. However, when investigating administration of specific agents, including antipsychotics and sedatives, given to delirious patients during ICU stay, mixed delirium received more agents compared to the other motor subtypes. These findings are complex and generate multiple considerations. Antipsychotics are supported as potentially beneficial in the treatment of hyperactive delirium [20]. It could be speculated that hyperactive delirium is more reversible by responding well to treatment resulting in shorter delirium duration, ICU stay, and reduced exposure to pharmacological interventions in general. All the investigated agents could induce a hypoactive state in an initially hyperactive patient, resulting in a medically induced mixed motor subtype. The longer ICU stay reported in patients with mixed delirium, compared to the other motor subtypes, may account for some of the increased antipsychotic and sedative exposure; however, the inverse relationship is also plausible as inappropriate treatment may complicate ICU stay causing prolonged delirium duration and death. Deep sedation, agitation, and cumulative dose of benzodiazepines have been associated with increased mortality [34].

Studies in our review rated low risk of bias or good quality had a higher proportion of patients with mixed delirium. This could be due to the methodology of delirium assessment strategy used in these studies, as studies screening for delirium several times daily and for a longer period were rated higher quality and patients in these studies would have greater chance of getting both hypo- and hyperactive delirium assessments. Studies in our review screened patients for delirium averagely 1.7 times a day, which is less than the recommended $2-3$ times daily (once every shift or $8 / 12 \mathrm{~h}$ ) [20]. Delirium is a transient condition so when delirium screening does not occur frequently one might miss hyper- or hypoactive periods in a delirious patient, hence misdiagnosing the delirium motor subtype. If mixed delirium is more common in studies that have increased assessments, then it is likely more common than most of the literature would suggest. However, with current knowledge it is impossible to determine whether mixed delirium is its own pathologic entity or simply present in patients experiencing disease entities of both hypoactive and hyperactive delirium combined.

\section{Strengths and limitations}

We included both RCTs and observational studies, which enabled us to investigate and describe the distribution of delirium motor subtypes in ICU patients independent of study design. Although making comparisons more difficult, this strengthens data quality and furthermore resulted in including the largest number of studies and largest pooled cohort evaluating distribution and outcomes of delirium motor subtypes to date.

This review also has limitations. First, heterogeneity in methodology and in outcome reporting amongst included studies was frequent. Second, not all studies differentiated between incidence and prevalence of delirium. Some studies used the term 'incidence of delirium' but did not account for having excluded patients with onset of delirium before ICU admission. Third, different methods for screening and diagnosis of delirium were applied across studies, and even though our review concludes that most studies applied CAM-ICU to diagnose delirium, the tool was not always used identically. Fourth, $74.1 \%$ of the included studies suffered from poor or unclear quality making baseline risk for confounding high at the point of data collection. Fifth, research on delirium motor subtypes mainly report low prevalence of pure hyperactive delirium. This is reflected in the wide confidence intervals surrounding ICU and hospital mortality in the hyperactive delirium cohort. Sixth, some studies defined mixed delirium as having both hypo- and hyperactive delirium assessments during the same day. Unless the authors could provide data on distribution of delirium motor subtype as defined in present review, the study was excluded. Seventh, propofol is often used to sedate agitated delirious patients and hence patients with hyperactive delirium. Propofol was unlikely used as delirium treatment but rather as part of a sedation strategy in hypoactive cases.

\section{Perspective}

It is important to clarify whether critically ill patients benefit from delirium motor subtype targeted treatments. Existing literature on outcomes related to delirium motor subtypes is poor and of low quality. Further studies are needed to determine whether short- and long-term outcomes are dependent on delirium motor subtypes and whether the subtypes react differently to treatments. Additionally, research on delirium motor subtypes in the ICU needs more strict standardization of exactly how, how often, and for how long a period delirium screening should occur. Since it is uncertain whether mixed delirium is truly its own disease entity, or just a mix hypoactive and hyperactive delirium episodes, future studies should consider time spent in each 
subtype for each patient, to examine whether one or the other is associated with worse outcomes.

\section{Conclusions}

In this review on distribution of delirium motor subtypes in the ICU, we found that hypoactive delirium was the most prevalent delirium motor subtype accounting for approximately half of delirium cases. Patients with mixed delirium had longer delirium duration, ICU and hospital length of stay, and higher ICU and hospital mortality than the other delirium motor subtypes. Patients with mixed delirium were more likely to receive administration of antipsychotics, $\alpha 2$-agonists, benzodiazepines, and propofol while in the ICU (no indication reported), while patients with hyperactive delirium were more likely to be intervened with a delirium-targeted pharmacological strategy. The identified differences among delirium motor subtypes in the ICU should be further investigated as they could be the key to future improvement of delirium care. The large between-study heterogeneity suggests that a more standardized methodology in delirium research is warranted.

\begin{abstract}
Abbreviations
APACHE: Acute Physiology and Chronic Health Evaluation; CAM-ICU: The Confusion Assessment Method for the ICU; DSM: Diagnostic and Statistical Manual for Mental Disorders; ICDSC: Intensive Care Delirium Screening Checklist; ICU: Intensive care unit; NIH: National Institutes of Health; PRISMA-SCR: Preferred Reporting Items for Systematic reviews and Meta-Analyses extension for Scoping Reviews; QA: Quality assessment; RASS: Richmond Agitation and Sedation Scale; RCT: Randomized controlled trial; SAPS: Simplified Acute Physiology Score; SOFA: Sequential Organ Failure Assessment.
\end{abstract}

\section{Supplementary Information}

The online version contains supplementary material available at https://doi. org/10.1186/s13054-022-03931-3.

Additional file 1. Search strings; Table S1. Overview of included studies; List of extracted data; Forrest plots: Fig. S1. Delirium incidence, Fig. S2. Delirium prevalence, Fig. S3. Distribution of hypoactive delirium, Fig. S4. Distribution of hyperactive delirium, Fig. S5. Distribution of mixed delirium; Table S2. Delirium assessment methods; Table S3. Characteristics of total cohort. Description of data. The data in the Additional file provides the reader with detailed search strings, a table with an overview of included studies with main characteristics and a reference for each study, a list of data we intended to extract for each study, forest plots of incidence and prevalence delirium and distribution of hypoactive, hyperactive and mixed delirium, a table showing data regarding delirium assessment methods used in included studies, and a table showing characteristics of the total pooled cohort in studies included.

\section{Acknowledgements}

Thanks to Penille Pless (Librarian) for helping with the systematic search strategy and to Birka Ravnholt Damlund (MD student) for helping with the screening process.
Authors' contributions

CKWK, SE, and NCA-R conceived the study. NCA-R and KNlaC wrote the protocol supervised by LMP and OM. KNlaC developed the search strategy together with a librarian and performed the literature search. KNlaC, NCA-R, SW, LMP, CBM, and MOC contributed to material preparation and data collection. KNlaC and NCA-R contributed to data analysis. Supervision was executed by OM who takes responsibility for the integrity of the work. The first draft of the manuscript was written by KlaC and NCA-R. All authors contributed to interpretation of data and critically revised the work. All authors read and approved the final manuscript.

\section{Funding}

No funding was received.

\section{Availability of data and materials}

The datasets used and/or analyzed during the current study are available from the corresponding author on reasonable request.

\section{Declarations}

Ethics approval and consent to participate

Consent was waived by the Ethics Committee in Region Zealand, Denmark, as reviews do not require ethical approval according to Danish law. Consent to participate was not applicable.

\section{Consent for publication}

Not applicable.

\section{Competing interests}

All authors declare no competing interests.

\section{Author details}

${ }^{1}$ Department of Anesthesiology, Centre for Anaesthesiological Research, Zealand University Hospital, Koege, Denmark. ${ }^{2}$ Department of Intensive Care, Copenhagen University Hospital, Rigshospitalet, Denmark. ${ }^{3}$ Department of Clinical Medicine, Copenhagen University, Copenhagen, Denmark.

Received: 5 December 2021 Accepted: 16 February 2022

Published online: 03 March 2022

\section{References}

1. Girard TD, Pandharipande PP, Ely EW. Delirium in the intensive care unit. Crit Care. 2008;12(Suppl 3):1-9.

2. Salluh JIF, Wang H, Schneider EB, Nagaraja N, Yenokyan G, Damluji A, et al. Outcome of delirium in critically ill patients: systematic review and metaanalysis. Br Med J. 2015;350:h2538.

3. Lipowski ZJ. Transient cognitive disorders (delirium, acute confusional states) in the elderly. Am J Psychiatry. 1983;140(11):1426-36.

4. Desforges JF, Lipowski ZJ. Delirium in the elderly patient. N Engl J Med. 1989;320(9):578-82.

5. Liptzin B, Levkoff SE. An empirical study of delirium subtypes. Br J Psychiatry. 1992;161(6):843-5.

6. Girard TD, Thompson JL, Pandharipande PP, Brummel NE, Jackson JC, Patel MB, et al. Clinical phenotypes of delirium during critical illness and severity of subsequent long-term cognitive impairment: a prospective cohort study. Lancet Respir Med. 2018;6(3):168-70.

7. Mulkey MA, Hardin SR, Olson DM, Munro CL. Pathophysiology review: seven neurotransmitters associated with delirium. Clin Nurse Spec J Adv Nurs Pract. 2018;32(4):195-211.

8. Hshieh TT, Fong TG, Marcantonio ER, Inouye SK. Cholinergic deficiency hypothesis in delirium: a synthesis of current evidence. J Gerontol Ser A Biol Sci Med Sci. 2008;63(7):764-72.

9. Gunther ML, Morandi A, Ely EW. Pathophysiology of delirium in the intensive care unit. Crit Care Clin. 2008;24(1):45-65.

10. Hughes C, Pandharipande P, Ely EW. Delirium: acute brain dysfunction in the critically ill, vol. 1. Cham: Springer; 2020. p. 209-17. 
11. Siddiqi N, House AO, Holmes JD. Occurrence and outcome of delirium in medical in-patients: a systematic literature review. Age Ageing. 2006;35(4):350-64.

12. Zhang Z, Pan L, Ni H. Impact of delirium on clinical outcome in critically ill patients: a meta-analysis. Gen Hosp Psychiatry. 2013;35(2):105-11.

13. Pandharipande PP, Girard TD, Jackson JC, Morandi A, Thompson JL, Pun BT, et al. Long-term cognitive impairment after critical illness. N Engl J Med. 2013;369(14):1306-16.

14. Meagher DJ, Leonard M, Donnelly S, Conroy M, Adamis D, Trzepacz PT. A longitudinal study of motor subtypes in delirium: relationship with other phenomenology, etiology, medication exposure and prognosis. J Psychosom Res. 2011;71(6):395-403.

15. Krewulak KD, Stelfox HT, Ely EW, Fiest KM. Risk factors and outcomes among delirium subtypes in adult ICUs: a systematic review. J Crit Care. 2020;56:257-64.

16. Rengel KF, Hayhurst CJ, Jackson JC, Boncyk CS, Patel MB, Brummel $\mathrm{NE}$, et al. Motoric subtypes of delirium and long-term functional and mental health outcomes in adults after critical illness. Crit Care Med. 2021;49(5):e521-32.

17. Meagher D. Motor subtypes of delirium: past, present and future. Int Rev Psychiatry. 2009;21(1):56-73.

18. Grover S, Sharma A, Aggarwal M, Mattoo SK, Chakrabarti S, Malhotra $S$, et al. Comparison of symptoms of delirium across various motoric subtypes. Psychiatry Clin Neurosci. 2014;68(4):283-91.

19. Tricco AC, Lillie E, Zarin W, O'Brien KK, Colquhoun H, Levac D, et al. Preferred reporting items for systematic reviews and meta-analyses extension for scoping reviews (PRISMA-ScR) checklist. Ann Intern Med. 2018;169(7):467-73.

20. The Society of Critical Care Medicine (SCCM). Clinical practice guidelines for the prevention and management of pain, agitation/sedation, delirium, immobility, and sleep disruption in adult patients in the ICU. Anesthesiology. 2018;46:e825-73.

21. Collet MO, Caballero J, Sonneville R, Bozza FA, Nydahl P, Schandl A, et al. Prevalence and risk factors related to haloperidol use for delirium in adult intensive care patients: the multinational AID-ICU inception cohort study. Intensive Care Med. 2018;44(7):1081-9.

22. Higgins JPT, Altman DG, Gøtzsche PC, Jüni P, Moher D, Oxman AD, et al. The Cochrane Collaboration's tool for assessing risk of bias in randomised trials. Br Med J. 2011;343:d5928.

23. National Heart, Lung, and Blood Institute. Study quality assessment tools; 2019. https://www.nhlbi.nih.gov/health-topics/study-quality-asses sment-tools.

24. Shi J, Luo D, Weng H, Zeng XT, Lin L, Chu H, et al. Optimally estimating the sample mean and standard deviation from the five-number summary. Res Synth Methods. 2020;11(5):641-54

25. Luo D, Wan X, Liu J, Tong T. Optimally estimating the sample mean from the sample size, median, mid-range, and/or mid-quartile range. Stat Methods Med Res. 2018;27(6):1785-805.

26. Higgins JPT, Thompson SG, Deeks JJ, Altman DG. Measuring inconsistency in meta-analyses. Br Med J. 2003;327:557-60.

27. R Core Team. R: a language and environment for statistical computing Vienna: R Foundation for Statistical Computing; 2019.

28. Krewulak KD, Stelfox HT, Leigh JP, Ely EW, Fiest KM. Incidence and prevalence of delirium subtypes in an adult ICU: a systematic review and meta-analysis. Crit Care Med. 2018:46(12):2029-35

29. Rood PJT, van de Schoor F, van Tertholen K, Pickkers P, van den Boogaard M. Differences in 90-day mortality of delirium subtypes in the intensive care unit: a retrospective cohort study. J Crit Care. 2019;53:120-4.

30. Pisani MA, Kong SY, Kasl SV, Murphy TE, Araujo KL, Van Ness PH, et al. Days of delirium are associated with 1-year mortality in an older intensive care unit population. Am J Respir Crit Care Med. 2009;180(11):1092-7.

31. Hughes CG, Hayhurst CJ, Pandharipande PP, Shotwell MS, Feng X, Wilson $J$ E, et al. Association of delirium during critical illness with mortality: multicenter prospective cohort study. Anesth Analg. 2021;133(5):1152-61.

32. van Velthuijsen EL, Zwakhalen SMG, Mulder WJ, Verhey FRJ, Kempen GIJM. Detection and management of hyperactive and hypoactive delirium in older patients during hospitalization: a retrospective cohort study evaluating daily practice. Int J Geriatr Psychiatry. 2018;33(11):1521-9.

33. Mac Sweeney R, Barber V, Page V, Ely EW, Perkins GD, Young JD, et al. A national survey of the management of delirium in UK intensive care units. QJM. 2010;103(4):243-51.
34. Aragón RE, Proaño A, Mongilardi N, De Ferrari A, Herrera P, Roldan R, et al. Sedation practices and clinical outcomes in mechanically ventilated patients in a prospective multicenter cohort. Crit Care. 2019;23(1):1-9.

\section{Publisher's Note}

Springer Nature remains neutral with regard to jurisdictional claims in published maps and institutional affiliations.
Ready to submit your research? Choose BMC and benefit from:

- fast, convenient online submission

- thorough peer review by experienced researchers in your field

- rapid publication on acceptance

- support for research data, including large and complex data types

- gold Open Access which fosters wider collaboration and increased citations

- maximum visibility for your research: over $100 \mathrm{M}$ website views per year

At BMC, research is always in progress.

Learn more biomedcentral.com/submissions 